\title{
Method Research for Signal Extraction of Noise Frequency Based on
}

\section{Modal Decomposition}

\author{
GeZhen ${ }^{1}$, LiuZhigang ${ }^{2, a}$ \\ ${ }^{1}$ School of Information Engineering,Wuhan Technology and Business University, Wuhan 430200, \\ China; \\ ${ }^{2}$ Nanchang Institute of Science\& Technology,Nanchang 330108,China \\ a1318747794@qq.com
}

\begin{abstract}
Keywords: Empirical mode decomposition (EMD); Collection of empirical mode decomposition (EEMD); Unascertained signal frequency
\end{abstract}

\begin{abstract}
In this article, it is proposed by using the empirical mode decomposition and the collection of empirical mode decomposition to decompose the signal and white noise to avoid patterns confused ideas of known frequency signal extraction method .In order to solve the known frequency signal extraction method, some of the problems faced in the extremum point positioning, such as the determination of amplitude, joining the white noise variance, and so on.
\end{abstract}

\section{Introduction}

Signal extraction technology is signal processing and an important part of modern information theory, is one of the pillars of the information technology. Is closely related to the extraction technology and scientific research and engineering practice, there are a lot of noise when the system, the relatively weak amplitude frequency of signal will be useless noise drown, know how to get from the system frequency signal of the phase and amplitude, is a difficult problem to be solved. For a long time, the Fourier transform is an important technology in signal processing, but as the multiplicity of the signal characteristic and the limitations of Fourier transform, the decomposition method for non-stationary signal. For example, empirical mode decomposition (EMD), collection of empirical mode decomposition (EEMD) zero space tracking algorithm [3] (NSP) and so on, these methods make up for the traditional signal processing in the disadvantages of the non-stationary signal. In this paper, through draw lessons from the thinking of EMD and EEMD decomposition signal, using the known frequency, improve and write a known frequency signal extraction method.

\section{Empirical mode decomposition and collection of empirical mode decomposition}

Norton 1998 Huang EMD (empirical mode decomposition) method is proposed, by extracting the average signal envelope of the up and down repeatedly screening process, get a series of time-domain adaptive to local symmetry and instantaneous frequency has a clear physical meaning of the IMF (the intrinsic mode function) signal. The EEMD [2] (Ensemble Empirical Mode Decomposition) method of the principle is the use of the Gaussian white noise with statistical characteristics of frequency distribution, when after adding white noise signal, will make the signal in different scales with connected - to minimize encountered in the process of the EMD Decomposition of modal aliasing. EEMD specific decomposition steps are as follows: 
Step 1: join in the initial signal $x(t)$ with mean zero many times, variance for constant white noise $n_{i}(t)$ I.e. $x_{i}(t)=x(t)+n_{i}(t), x_{i}(t)$ in the type is the first time $i$ to join Gaussian white noise signal.

Step 2: to the EMD decomposition of signals $x_{i}(t)$, the IMF component for $c_{i j}(t)$, said after the first time to join Gaussian white noise, a decomposition of the first component of the IMF.

Step 3: repeat the step 1 and step 2, the corresponding IMF component to the overall average operation, eliminate the influence of multiple white Gaussian noise was added into the real, the resulting EEMD after decomposition of the IMF components as:

$$
c_{j}(t)=\frac{1}{N} \sum_{i=1}^{N} c_{i j}(t)
$$

In the formula $(1), c_{j}(t)$ is the $\mathrm{j}$-th IMF component after the initial signal EEMD decomposition. When the number of repeat $\mathrm{N}$, the greater the corresponding IMF component of Gaussian white noise and tends to zero, the EEMD decomposition results as follows:

$$
x(t)=\sum_{j} c_{j}(t)+r(t)
$$

Set $x(t)$ consists of Gaussian white noise by $N(t)$ and the frequency known signal $S(t)=\cos \left(2 \pi f_{n} N\right), f_{n}=0.001$, the signal-to-noise ratio is $0 \mathrm{db}$. The Partial IMF component which from $x(t)$ after EEMD algorithm are shown in Fig.1 below:


Fig.1 Partial results of EEMD decomposition of $x(t)$

The eighth IMF component of EEMD decomposition is the closest to the frequency known signal after extracted, for better fitting frequency signal, the three IMF components in figure 1 combined compared with known frequency signal, which are shown in Fig.2 below: 

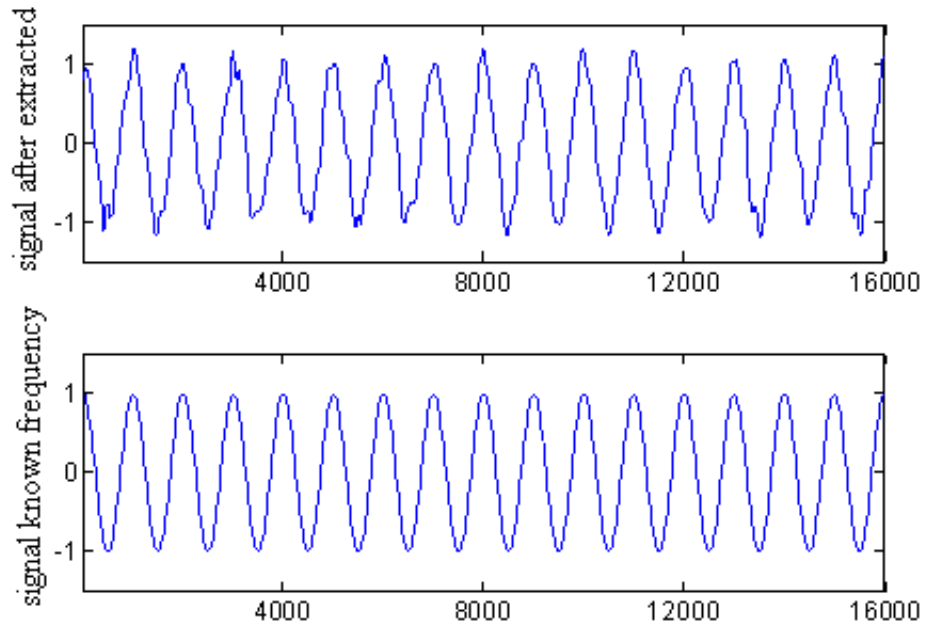

Fig.2 Part of the IMF combination signal and frequency deterministic signal

From figure 2 shows that even if the parts together to get the combination of the signal and the frequency of known signal there is still a larger error.

\section{The extraction of frequency known signal under noise}

The known frequency signal extraction method is to use the EEMD filtering of signal and white noise and the EMD thought [4-7], steps are as follows:

Step 1: determine the signal $x_{i}(t)$ all the local extremum points;

Step 2: select the extreme value point to meet the extreme value point interval and the amplitude of known frequency signal is are larger in all local extremum points;

Step 3: use the method of interpolation of envelope structure, and using the average envelope envelope and the up and down;

Step 4: join with mean zero, variance for constant white noise $n_{i}(t)$, repeat steps $1,2,3$, every time $m_{i}(t)$ to get up, after the average get known frequency signal; The above method used to extract $x(t)$, the result as shown in the Fig.3 below:
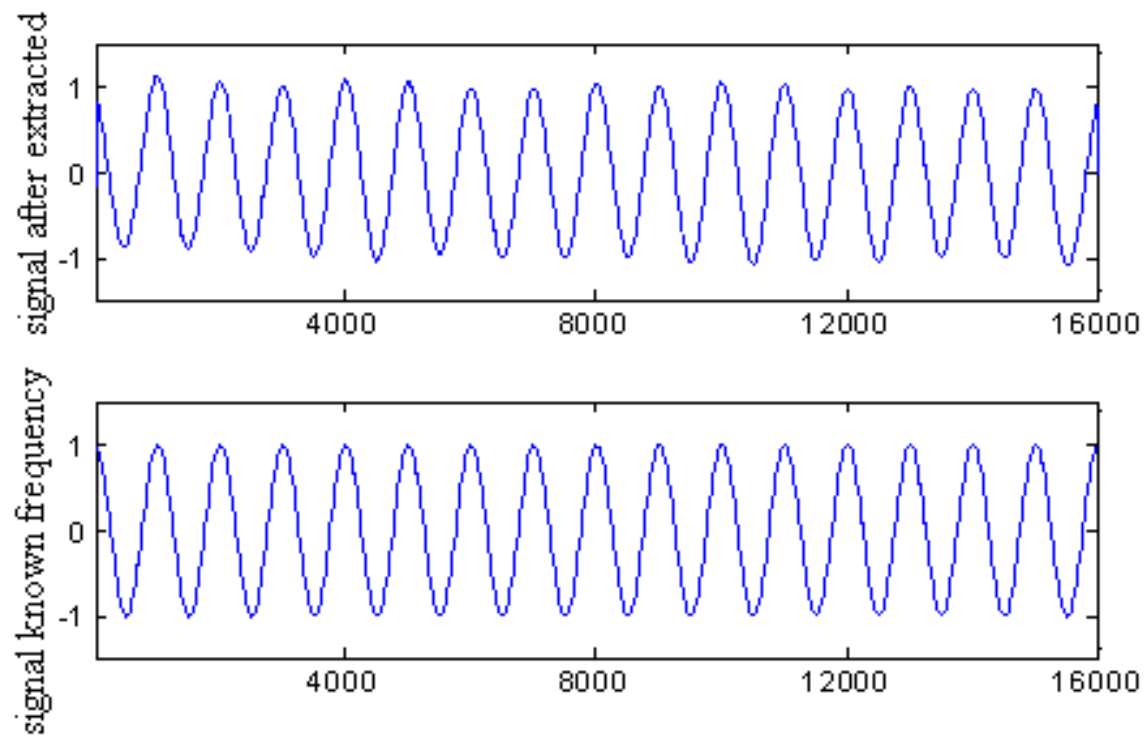

Fig. 3 The extracted signal and frequency deterministic signal

Comparing figure 2 and figure 3, we can see that using noise frequency under the known signal extraction method extracted signal closer to the frequency of the signal $S(t)$. 


\section{Conclusion}

EMD and EEMD are widely used in signal processing.This paper draw lessons from the thinking of the decomposed signal, and proposes a frequency of signal extraction method under noise, which verified the method of the signal extracted into frequency white noise can be well orientation assisted know extreme value point of the signal, the effectiveness of the proposed method is validated by computer simulation.

\section{Acknowlegement}

The work was supported by the scientific research project of Wuhan Technology and Business University with the project number A2015005 and the project name Design of defect detection system for porous ceramic structure based on multi frequency eddy current and machine vision.

\section{References}

[1] N. E. Huang, Z. Shen, and S. R. Long, "The empirical mode decomposi-tion and the Hilbert pectrum for nonlinear and nonstationary time series analysis," Proc. R. Soc. London. A, 1998, 454: 903-995.

[2] Z.H. Wu, N.E. Huang, "Ensemble empirical mode decomposition: a noise-assisted data analysis method," Advances in Adaptive Data Analysis, 2009, 1, doi: 10.1142/S1793536909000047:1-41

[3] Peng Silong,Hwang Wen-Liang Null Space Pursuit An Operator-based Approach to Adaptive ignal Separation.IEEE Trans Signal Processing 2010,58(5): 2475-2483.

[4] Hong Hong, Xinlong Wang, Zhiyong Tao "Local Integral Mean-Based Sifiting for Empirical Mode Decomposition,” IEEE Signal Processing Letters, 2009, 16 (10): 841-844,

[5] Qiong Xie, Jiangping Li, Xiaoguang Gao "Real time infrared gas detection based on a modified EMD algorithm," Sensors and Actuators B .2009: 303-309.

[6] G. Rilling and P. Flandrin, "One or two frequencies? the emd answers,"IEEE transactions on signal processing, 2008, 56: 85-95.

[7] Rongjun Lu,Bin Zhou,Wei Gao On Signal Decomposition Performance Of Null Space Pursuit International Conference on Intelligent Control and Information Processing August 13-15,2010-Dalian, China. 